\title{
High-order non-reflecting boundary conditions for dispersive waves
}

\author{
Dan Givoli $^{\mathrm{a}, *, 1}$, Beny Neta ${ }^{\mathrm{b}, 2}$ \\ a Department of Aerospace Engineering, and Asher Center for Space Research \\ Technion-Israel Institute of Technology, Haifa 32000, Israel \\ b Department of Mathematics, Naval Postgraduate School, 1141 Cunningham Road, Monterey, CA 93943, USA
}

Received 3 April 2002; received in revised form 17 July 2002; accepted 1 August 2002

\begin{abstract}
Problems of linear time-dependent dispersive waves in an unbounded domain are considered. The infinite domain is truncated via an artificial boundary $\mathcal{B}$, and a high-order non-reflecting boundary condition (NRBC) is imposed on $\mathcal{B}$. Then the problem is solved by a finite difference (FD) scheme in the finite domain bounded by $\mathcal{B}$. The sequence of NRBCs proposed by Higdon is used. However, in contrast to the original low-order implementation of the Higdon conditions, a new scheme is devised which allows the easy use of a Higdon-type NRBC of any desired order. In addition, a procedure for the automatic choice of the parameters appearing in the NRBC is proposed. The performance of the scheme is demonstrated via numerical examples. (C) 2002 Elsevier Science B.V. All rights reserved.
\end{abstract}

\section{Introduction}

In many fields of application involving the propagation of waves, the domain of the problem under investigation is unbounded (or very large). One of the common methods used for the numerical solution of such problems [1] is the method of non-reflecting boundary conditions (NRBCs). In this method the original domain is truncated via an artificial boundary $\mathcal{B}$, thus forming a finite computational domain $\Omega$ bounded by $\mathcal{B}$. A special boundary condition is imposed on $\mathcal{B}$, in order to complete the statement of the problem (i.e., make the solution unique) and, most importantly, to ensure that no (or little) spurious wave reflection occurs from $\mathcal{B}$. Then the problem is solved numerically in $\Omega$.

The boundary condition applied on $\mathcal{B}$ is called a NRBC, although a few other names are often used too [2]. Naturally, the quality of the numerical solution strongly depends on the properties of the NRBC employed. In the last 25 years or so, much research has been done to develop NRBCs that after discretization lead to a scheme which is stable, accurate, efficient and easy to implement. See [3,4] for recent reviews on the subject. Of course, it is difficult to find a single NRBC which is ideal in all respects and all cases; this is why the quest for better NRBCs and their associated discretization schemes continues.

\footnotetext{
* Corresponding author.

E-mail addresses: givolid@aerodyne.technion.ac.il (D. Givoli), bneta@ nps.navy.mil (B. Neta).

1 This work has been done while the author was on leave at the Naval Postgraduate School, Monterey, CA.

2 Tel.: +1-831-656-2235; fax: +1-831-656-2355.
} 
Some low-order local NRBCs have been proposed in the late 1970s and early 1980s and have become well-known, e.g., the Engquist-Majda NRBCs [5] and the Bayliss-Turkel NRBCs [6]. The late 1980s and early 1990s have been characterized by the emerging of the exact non-local Dirichlet-to-Neumann (DtN) NRBC [7,8] and the perfectly matched layer (PML) [9]. More recently, high-order local NRBCs have been introduced. Sequences of increasing-order NRBCs have been available before (e.g., the Bayliss-Turkel [6] conditions constitute such a sequence), but they had been regarded as impractical beyond 2nd or 3rd order from the implementation point of view. Only since the mid-1990s, practical high-order NRBCs have been devised.

The first such high-order NRBC has apparently been proposed by Collino [10], for two-dimensional time-dependent waves in rectangular domains. Its construction requires the solution of the one-dimensional wave equation on $\mathcal{B}$. Grote and Keller [11] developed a high-order converging NRBC for the three-dimensional time-dependent wave equation, based on spherical harmonic transformations. They extended this NRBC for the case of elastic waves in [12]. Sofronov [13] has independently published a similar scheme in the Russian literature. Hagstrom and Hariharan [14] constructed high-order NRBCs for the two- and three-dimensional time-dependent wave equations based on the analytic series representation for the outgoing solutions of these equations. It looks simpler than the previous two NRBCs. For time-dependent waves in a two-dimensional waveguide, Guddati and Tassoulas [15] devised a high-order NRBC by using rational approximations and recursive continued fractions. Givoli [16] has shown how to derive high-order NRBCs for a general class of wave problems, leading to a symmetric finite element formulation. In [17], this methodology was applied to the particular case of time-harmonic waves, using optimally localized DtN NRBCs.

Most of the NRBCs mentioned above have been designed for either time-harmonic waves or for non-dispersive time-dependent waves. The presence of wave dispersion makes the time-dependent problem much more difficult as far as NRBC treatment is concerned. Dispersive media appear in various applications. One important example is that of meteorological models which take into account the earth rotation [18]. Other examples include quantum-mechanics waves, the vibration of structures with rotational rigidity such as beams, plates and shells, and many non-linear wave problems, with or without linearization. Very recently, Navon et al. [19] developed a PML scheme for the dispersive shallow water equations (SWEs). In the present paper we develop high-order NRBCs for dispersive waves. Naturally, our scheme is just as applicable to the non-dispersive case, by simply taking the dispersion parameter to be zero.

Higdon [20] proposed a sequence of NRBCs for the dispersive (Klein-Gordon) wave equation. In fact, these NRBCs were developed originally for non-dispersive waves [21-25], but Higdon showed in [20] that they can be applied in the dispersive case too. Indeed, our scheme is based on Higdon's NRBCs. However, in contrast to the original low-order formulation of these conditions, a new scheme is devised here which allows the easy use of a Higdon-type NRBC of any desired order. In addition, a procedure for the automatic choice of the parameters appearing in the NRBC is proposed.

Following is the outline of the rest of this paper. In Section 2 we state the problem under investigation. We emphasize that the setup taken here, namely that of a semi-infinite waveguide, serves merely as an example, and in fact the proposed approach is general and can easily be extended to other configurations, such as two-dimensional exterior problems with a rectangular artificial boundary. In Section 3 we present the Higdon NRBCs and briefly recall their properties. In Section 4 we show how to implement the Higdon NRBCs, using a finite difference (FD) scheme, in a high-order way. Then we show, in Section 5, how the basic FD approximations used in this scheme can be improved if one desires, and in Section 6 we present a procedure for the automatic choice of the NRBC parameters. We demonstrate the performance of the new method via some numerical examples in Section 7. We conclude, in Section 8, with some remarks.

\section{Statement of the problem}

We consider the linear inhomogeneous Klein-Gordon equation

$$
\partial_{t}^{2} u-C_{0}^{2} \nabla^{2} u+f^{2} u=S
$$




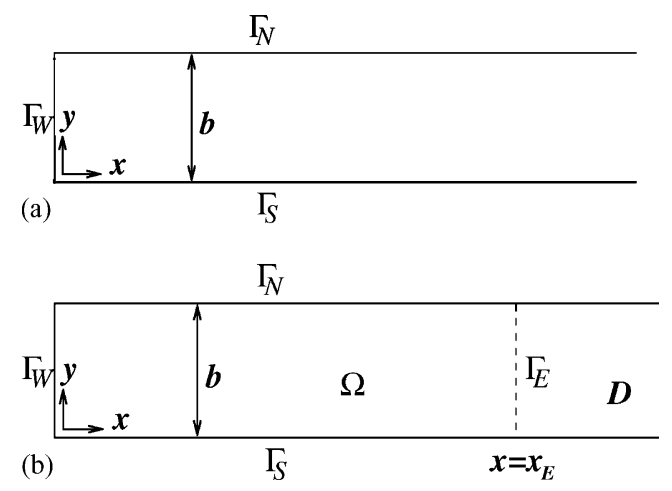

Fig. 1. Setup for the waveguide problem: (a) the original problem in a semi-infinite waveguide; (b) the computational problem in a finite domain $\Omega$.

in a two-dimensional uniform semi-infinite channel or waveguide. A Cartesian coordinate system $(x, y)$ is introduced such that the waveguide is parallel to the $x$-direction. The width of the waveguide is denoted as $b$. The setup is shown in Fig. 1(a). In (1), $u$ is the unknown wave field, $C_{0}$ is the given reference wave speed, $f$ is the given dispersion parameter and $S$ is a given wave source function. The $C_{0}$ and $f$ are allowed to be functions of location; however, it is assumed that the region where they are not constant is finite (and typically located near the west boundary $\Gamma_{\mathrm{W}}$ ). The wave source $S$ is a function of location and time, but it is assumed to have a local support. Eq. (1) describes, for example, the lateral vibration of a membrane strip on an elastic foundation, or the acoustic pressure in a dispersive medium (say, a linearized bubbly medium). Also, it can be shown that the linearized SWEs, with a flat bottom and zero initial conditions, reduce to (1), where $u$ is the water elevation above the reference level [18]. In the geophysical context, $f$ is called the Coriolis parameter and is related to the angular velocity of the earth.

On the south and north boundaries $\Gamma_{\mathrm{S}}$ and $\Gamma_{\mathrm{N}}$ we specify the Neumann condition:

$$
\partial_{y} u=0 \quad \text { on } \Gamma_{\mathrm{S}} \text { and } \Gamma_{\mathrm{N}} .
$$

In acoustics this corresponds to a "hard wall" condition. On the west boundary $\Gamma_{\mathrm{W}}$ we prescribe $u$ using a Dirichlet condition, i.e.

$$
u(0, y, t)=u_{\mathrm{W}}(y, t) \quad \text { on } \Gamma_{\mathrm{W}},
$$

where $u_{\mathrm{W}}(y, t)$ is a given function (incoming wave). At $x \rightarrow \infty$ the solution is known to be bounded and not to include any incoming waves.

To complete the statement of the problem, the initial conditions

$$
u(x, y, 0)=u_{0}, \quad \partial_{t} u(x, y, 0)=v_{0}
$$

are given at time $t=0$ in the entire domain. We assume that the functions $u_{0}$ and $v_{0}$ have a local support.

We now truncate the semi-infinite domain by introducing an artificial east boundary $\mathcal{B} \equiv \Gamma_{\mathrm{E}}$, located at $x=x_{\mathrm{E}}$. See Fig. 1(b). This boundary divides the original semi-infinite domain into two subdomains: an exterior domain $D$, and a finite computational domain $\Omega$ which is bounded by $\Gamma_{\mathrm{W}}, \Gamma_{\mathrm{N}}, \Gamma_{\mathrm{S}}$ and $\Gamma_{\mathrm{E}}$. We choose the location of $\Gamma_{\mathrm{E}}$ such that the entire support of $S, u_{0}$ and $v_{0}$ and the region of non-uniformity of $C_{0}$ and $f$ are all contained inside $\Omega$. Thus, on $\Gamma_{\mathrm{E}}$ and in $D$, the homogeneous counterpart of (1) holds, i.e.

$$
\partial_{t}^{2} u-C_{0}^{2} \nabla^{2} u+f^{2} u=0
$$

with constant coefficients $C_{0}$ and $f^{2}$, and the medium is initially at rest.

To obtain a well-posed problem in the finite domain $\Omega$ we need to impose a boundary condition on $\Gamma_{\mathrm{E}}$. This must be a NRBC, so as to prevent spurious reflection of waves. In the next section we discuss the choice of this NRBC. 


\section{Higdon's NRBCs}

On the artificial boundary $\Gamma_{\mathrm{E}}$ we use one of the Higdon NRBCs [20]. These NRBCs were presented and analyzed in a sequence of papers [21-25] for non-dispersive acoustic and elastic waves, and were extended in [20] for the dispersive case. The Higdon NRBC of order $J$ is

$$
H_{J}:\left[\prod_{j=1}^{J}\left(\partial_{t}+C_{j} \partial_{x}\right)\right] u=0 \quad \text { on } \Gamma_{\mathrm{E}} .
$$

Here, the $C_{j}$ are parameters which have to be chosen and which signify phase speeds in the $x$-direction. The main advantages of the Higdon conditions are as follows:

- The Higdon NRBCs are very general, namely they apply to a variety of wave problems, in one, two and three dimensions and in various configurations. Moreover, they can be used, without any difficulty, for dispersive wave problems and for problems with layers. Most other available NRBCs are either designed for non-dispersive homogeneous media (as in acoustics and electromagnetics) or are inherently of low order (as in meteorology and oceanography).

- The Higdon NRBCs constitute a sequence of conditions of increasing order. This, and the fact that no asymptotic approximation is involved in their construction, enables one in principle (leaving implementational issues aside for the moment) to obtain solutions with unlimited accuracy.

- For certain choices of the parameters, the Higdon NRBCs are equivalent to NRBCs that are derived from rational approximation of the dispersion relation (the Engquist-Majda conditions [5] being the most well-known example). This has been proved by Higdon in [20,21]. More precisely, Higdon's theorem states that if a NRBC is based on a symmetric rational approximation to the dispersion relation corresponding to outgoing waves, then it is either (a) equivalent to (6) for a suitable choice of $J$ and the parameters $C_{j}$, or (b) unstable, or (c) not optimal. Lack of optimality means here that the coefficients in the NRBC can be modified so as to reduce the amount of the spurious reflection. Thus, the Higdon NRBCs can be viewed as generalization of rational approximation NRBCs.

The scheme developed here is different than the original Higdon scheme [20] in the following ways:

(a) The discrete Higdon conditions were developed in the literature up to third order only, because of their algebraic complexity which increases rapidly with the order. Here we show how to easily implement these conditions to an arbitrarily high-order. The scheme is coded once and for all for any order; the order of the scheme is simply an input parameter.

(b) The Higdon NRBCs involve some parameters which must be chosen. Higdon [20] discusses some general guidelines for their manual a priori choice by the user. We shall show how these parameters can be chosen automatically.

(c) We shall show how to improve the discretization of the basic operators involved in the Higdon NRBCs, by using more accurate FD stencils, and how to incorporate these improved discretizations in the new scheme.

We now make a few remarks recalling the properties of the Higdon NRBCs:

1. The boundary condition (6) is exact for all waves that propagate with an $x$-direction phase speed equal to either of $C_{1}, \ldots, C_{J}$. To see this, consider a wave which satisfies the wave equation (5) and boundary condition (2) with constant $C_{0}$ and $f$. Such a wave has the form

$$
u=A \cos \frac{n \pi y}{b} \cos (k x-\omega t+\psi),
$$

where

$$
\omega^{2}=C_{0}^{2}\left(k^{2}+\frac{n^{2} \pi^{2}}{b^{2}}\right)+f^{2}, \quad n=0,1,2, \ldots .
$$


Also, let

$$
C_{x}=\frac{\omega}{k},
$$

which is the $x$-direction phase velocity. In (7)-(9), $A$ is the wave amplitude, $\psi$ is its phase, $k$ is the $x$-component wavenumber, and $\omega$ is the wave frequency. Eq. (8) is the dispersion relation. In general, solutions of (5) consist of an infinite number of waves of the form (7). There are also solutions that decay exponentially in the $x$-direction; however, they are usually not of great concern, since the decaying modes are expected to be insignificant at the time they reach $\Gamma_{\mathrm{E}}$. Now, it is easy to verify that if one of the $C_{j}$ 's in (6) is equal to $C_{x}$, then the wave (7) satisfies the boundary condition (6) exactly.

2. From (8) and (9) we have

$$
C_{x}=\sqrt{C_{0}^{2}+\frac{C_{0}^{2} n^{2} \pi^{2} / b^{2}+f^{2}}{k^{2}}}, \quad n=0,1,2, \ldots .
$$

Thus, always $C_{x} \geq C_{0}$, hence one should take $C_{j} \geq C_{0}$.

3. The first-order condition $H_{1}$ is a Sommerfeld-like boundary condition. If we set $C_{1}=C_{0}$ we get the classical Sommerfeld-like NRBC. A lot of work in the meteorological literature is based on using $H_{1}$ with a specially chosen $C_{1}$. Pearson [26] used a special but constant value of $C_{1}$, while in the scheme devised by Orlanski [27] and in later improved schemes [28-31] the $C_{1}$ changes dynamically and locally in each time-step based on the solution from the previous time-step. Some of the limited-area weather prediction codes used today are based on such schemes, e.g., COAMPS [32]. See also the recent papers [33-35] where several such adaptive $H_{1}$ schemes are compared.

4. The condition $H_{J}$ involves normal and temporal derivatives up to $J$ th order. In fact, it has the form

$$
\sum_{j=0}^{J} w_{j} \partial_{x}^{j} \partial_{t}^{J-j} u=0
$$

which is obtained by expanding (6). Finding the general formula for the coefficients $w_{j}$ in (11) is difficult, but fortunately we shall not need it in our new scheme.

5. It is easy to show (see Higdon [20] for a similar setting) that when a wave of the form (7) impinges on the boundary $\Gamma_{\mathrm{E}}$ where the NRBC $H_{J}$ is imposed, the resulting reflection coefficient is

$$
R=\prod_{j=1}^{J}\left|\frac{C_{j}-C_{x}}{C_{j}+C_{x}}\right|
$$

Again we see that if $C_{j}=C_{x}$ for any one of the $j$ 's then $R=0$, namely there is no reflection and the NRBC is exact. Moreover, we see that the reflection coefficient is a product of $J$ factors, each of which is smaller than 1. This implies that the reflection coefficient becomes smaller as the order $J$ increases regardless of the choice made for the parameters $C_{j}$. Of course, a good choice for the $C_{j}$ would lead to better accuracy with a lower order $J$, but even if we miss the correct $C_{j}$ 's considerably (say, if we make the simplest choice $C_{j}=C_{0}$ for $j=1, \ldots, J)$, we are still guaranteed to reduce the spurious reflection as we increase the order $J$. This is an important property of the Higdon's NRBCs and is the reason for their robustness.

6. In [23], Higdon points to the possibility of a long-time instability that might occur when one uses a NRBC with high-order derivatives. If the interior governing equations and the NRBC both admit solutions at zero wavenumber and frequency, and if the data in the problem include such "zero modes", then a slowly growing smooth instability is possible. Whether this shows up in practice depends on the order of the derivatives in the NRBC and on the number of spatial dimensions. However, these difficulties do not arise in the presence of dispersion, or if the data are confined to non-trivial modes. 


\section{Discretization of Higdon's NRBCs}

The Higdon condition $H_{J}$ given by (6) is a product of $J$ operators of the form $\partial_{t}+C_{j} \partial_{x}$. Consider the following FD approximations:

$$
\partial_{t} \simeq \frac{I-S_{t}^{-}}{\Delta t}, \quad \partial_{x} \simeq \frac{I-S_{x}^{-}}{\Delta x} .
$$

In (13), $\Delta t$ and $\Delta x$ are, respectively, the time-step size and grid spacing in the $x$-direction, $I$ is the identity operator, and $S_{t}^{-}$and $S_{x}^{-}$are shift operators defined by

$$
S_{t}^{-} u_{p q}^{n}=u_{p q}^{n-1}, \quad S_{x}^{-} u_{p q}^{n}=u_{p-1, q}^{n} .
$$

Here and elsewhere, $u_{p q}^{n}$ (and also $\left.u_{p, q}^{n}\right)$ is the FD approximation of $u(x, y, t)$ at grid point $\left(x_{p}, y_{q}\right)$ and at time $t_{n}$. We use (13) into (6) to obtain

$$
\left[\prod_{j=1}^{J}\left(\frac{I-S_{t}^{-}}{\Delta t}+C_{j} \frac{I-S_{x}^{-}}{\Delta x}\right)\right] u_{E q}^{n}=0 .
$$

Here, the index $E$ correspond to a grid point on the boundary $\Gamma_{\mathrm{E}}$. Higdon has solved this difference equation (and also a slightly more involved equation that is based on time- and space-averaging approximations for $\partial_{x}$ and $\partial_{t}$; see next section) for $J \leq 3$ to obtain an explicit formula for $u_{E q}^{n}$. This formula is used to find the current values on the boundary $\Gamma_{\mathrm{E}}$ after the solution in the interior points and on the other boundaries has been updated; thus it is an explicit formula. The formula for $J=2$ is found in [25], and the one for $J=3$ appears in the appendix of [24]. The algebraic complexity of these formulas increases rapidly with the order $J$. It is thus not surprising that we have not found in the literature any report on the implementation of the Higdon NRBCs beyond $J=3$.

Now we show how to implement the Higdon NRBCs to any order using a simple algorithm. To this end, we first multiply (15) by $\Delta t$ and rearrange to obtain

$$
Z \equiv\left[\prod_{j=1}^{J}\left(a_{j} I+d_{j} S_{t}^{-}+e_{j} S_{x}^{-}\right)\right] u_{E q}^{n}=0,
$$

where

$$
\begin{aligned}
& a_{j}=1+\frac{C_{j} \Delta t}{\Delta x}, \\
& d_{j}=-1, \\
& e_{j}=-\frac{C_{j} \Delta t}{\Delta x} .
\end{aligned}
$$

The coefficient $d_{j}$ actually does not depend on $j$, but we keep this notation to allow easy extensions to the scheme (see next section). Now, $Z$ in (16) can be written as a sum of $3^{J}$ terms, each one is an operator acting on $u_{E q}^{n}$, namely

$$
Z \equiv \sum_{m=0}^{3^{J}-1} A_{m} P_{m} u_{E q}^{n}=0 .
$$

Here $A_{m}$ is a coefficient depending on the $a_{j}, d_{j}$ and $e_{j}$, and $P_{m}$ is an operator involving products of $I, S_{t}^{-}$and $S_{x}^{-}$. All the terms in the sum in (20) are computable at the current time-step $n$, except the one which involves only the identity operator and no shift operators. If we let this term correspond to $m=0$, then $P_{0}=I$ and

$$
A_{0}=\prod_{j=1}^{J} a_{j}
$$


Thus we get from (20)

$$
Z \equiv A_{0} u_{E q}^{n}+Z^{*}=0
$$

where

$$
Z^{*}=\sum_{m=1}^{3^{J}-1} A_{m} P_{m} u_{E q}^{n} .
$$

From (22) we get

$$
u_{E q}^{n}=-\frac{Z^{*}}{A_{0}},
$$

which is the desired value of $u$ on the boundary $\Gamma_{\mathrm{E}}$.

\section{Box 1. Algorithm for implementing the Higdon NRBC of order $J$.}

- Start with $Z^{*}=0$. Calculate $A_{0}=\prod_{j=1}^{J} a_{j}$.

- Loop over the integers $m=1, \ldots, 3^{J}-1$.

- For a given $m$, transform $m$ into a number $r$ in base 3, consisting of the digits 0,1 and 2 only. The length of $r$ will be at most $J$ digits. Store the $J$ digits of $r$ in the vector $D_{r}(j), j=1, \ldots, J$.

Example: Suppose that $J=6$ and $m=227$. Since 227 in base 3 is $r=22102$, we will get $D_{r}=$ $\left\{\begin{array}{llllll}0 & 2 & 2 & 1 & 0 & 2\end{array}\right\}$.

○ Use $D_{r}$ to calculate the coefficient $A_{m}$. To this end, start with $A_{m}=1$, loop over $j=1, \ldots, J$, and for each $j$ multiply $A_{m}$ by the factor $a_{j}$ (if $D_{r}(j)=0$ ) or $d_{j}$ (if $D_{r}(j)=1$ ) or $e_{j}$ (if $D_{r}(j)=2$ ).

Example: For $J=6$ and $m=227$, we have received the vector $D_{r}$ above. Then $A_{227}=a_{1} e_{2} e_{3} d_{4} a_{5} e_{6}$.

- Use $D_{r}$ to calculate the operator action $P_{m} u_{E q}^{n}$. To this end, start with $\hat{n}=n$ and $\hat{i}=E$, loop over $j=1, \ldots, J$, and for each $j$ subtract 1 from $\hat{n}$ (if $D_{r}(j)=1$ ) or subtract 1 from $\hat{i}$ (if $D_{r}(j)=2$ ) or do nothing (if $D_{r}(j)=0$ ). After the loop ends we have $P_{m} u_{E q}^{n}=u_{\hat{i} q}^{\hat{n}}$.

Example: For the case $J=6$ and $m=227$ considered above, we get $\hat{n}=n-1$ (because the digit "1" appears only once in $D_{r}$ ), and $\hat{i}=E-3$ (because the digit "2" appears three times in $D_{r}$ ). Hence $P_{227} u_{E q}^{n}=u_{E-3, q}^{n-1}$.

○ Update: $Z^{*} \leftarrow Z^{*}+A_{m} u_{\hat{i} q}^{\hat{n}}$.

- Next $m$.

- $u_{E q}^{n}=-Z^{*} / A_{0}$.

The problem now reduces to calculating $Z^{*}$ given by (23). We do this using the algorithm described in Box 1 . The basic idea is to calculate the coefficients $A_{m}$ and the operator actions $P_{m} u_{E q}^{n}$ term by term. This is done systematically by transforming the integer counter $m$ to a number in base 3 with $J$ digits. The $A_{m}$ and $P_{m}$ are not simple functions of the decimal representation of the number $m$, but they are simple functions of the digits of the base-3 representation of $m$.

Note that we need to store $u_{\hat{i} q}^{\hat{n}}$ values for $\hat{i}=E, E-1, \ldots, E-J$ and $\hat{n}=n, n-1, \ldots, n-J$. In other words, we have to store the history of the values of $u$ for a layer of thickness $J+1$ points near the boundary $\Gamma_{\mathrm{E}}$ and for $J+1$ time levels (including the current one). If there are $N_{y}$ grid points in the $y$-direction, then the amount of storage needed in a simple storage scheme is $(J+1)^{2} N_{y}$. However, one can save in storage by exploiting the fact that not all values $u_{\hat{i} q}^{\hat{n}}$ are needed, but only those for which $(E-\hat{i})+(n-\hat{n}) \leq J$. This is clear from (11) and also from (16). For example, the solution at time $t_{n-J}$ should be stored only for points on the boundary $\Gamma_{\mathrm{E}}$ itself. 
Higdon [20] has proved, in the context of the scalar Klein-Gordon equation (5), that the discrete NRBCs (15) are stable if the interior scheme is the standard second-order centered difference scheme

$$
\begin{aligned}
u_{p q}^{n+1}= & 2 u_{p q}^{n}-u_{p q}^{n-1}+\left(\frac{C_{0} \Delta t}{\Delta x}\right)^{2}\left(u_{p+1, q}^{n}-2 u_{p q}^{n}+u_{p-1, q}^{n}\right) \\
& +\left(\frac{C_{0} \Delta t}{\Delta y}\right)^{2}\left(u_{p, q+1}^{n}-2 u_{p q}^{n}+u_{p, q-1}^{n}\right)-(f \Delta t)^{2} u_{p q}^{n} .
\end{aligned}
$$

We use this interior scheme in the numerical experiments presented in Section 7. Since both (25) and the discretized Higdon NRBC are explicit, the whole scheme is explicit.

An alternative formulation of the Higdon NRBC, where all the high-order derivatives are eliminated by the use of auxiliary variables, is currently under development and will be reported in a future publication.

\section{Improved discrete Higdon NRBCs}

The discretization scheme described in the previous section is based on the basic FD approximations given by (13). These approximations can be improved in several ways. For example

(a) We can take

$$
\partial_{t} \simeq \frac{I-S_{t}^{-}}{\Delta t}\left((1-b) I+b S_{x}^{-}\right), \quad \partial_{x} \simeq \frac{I-S_{x}^{-}}{\Delta x}\left((1-b) I+b S_{t}^{-}\right),
$$

where $0 \leq b \leq 1$. Thus, the temporal difference is calculated with a weighted average in space while the spatial difference is calculated with a weighted averaged in time. The formulas (13) correspond to $b=0$. In [20], Higdon has used this approximation with $b=0.5$, and reported a slight improvement in the results compared to the use of (13).

(b) We can take one-sided approximations for the $x$ - and $t$-derivatives [36], i.e.

$$
\partial_{t} \simeq \frac{3 I-4 S_{t}^{-}+\left(S_{t}^{-}\right)^{2}}{2 \Delta t}, \quad \partial_{x} \simeq \frac{3 I-4 S_{x}^{-}+\left(S_{x}^{-}\right)^{2}}{2 \Delta x} .
$$

These approximations are second-order accurate, as opposed to those in (13) which are first-order accurate.

(c) We can combine the two types of approximations given above, namely

$$
\partial_{t} \simeq \frac{3 I-4 S_{t}^{-}+\left(S_{t}^{-}\right)^{2}}{2 \Delta t}\left((1-b) I+b S_{x}^{-}\right), \quad \partial_{x} \simeq \frac{3 I-4 S_{x}^{-}+\left(S_{x}^{-}\right)^{2}}{2 \Delta x}\left((1-b) I+b S_{t}^{-}\right) .
$$

The procedure described in the previous section for implementing the Higdon NRBCs can easily be modified to admit each of these improved approximations. The main feature that has to be changed in the algorithm outlined in Box 1 is the base to which the counting decimal integer $m$ is transformed. For example, consider the weighted approximation (26) replacing (13). In this case (16), which involves three basic operators $\left(I, S_{t}^{-}\right.$and $\left.S_{x}^{-}\right)$is replaced by

$$
Z \equiv\left[\prod_{j=1}^{J}\left(a_{j} I+d_{j} S_{t}^{-}+e_{j} S_{x}^{-}+g_{j} S_{t}^{-} S_{x}^{-}\right)\right] u_{E q}^{n}=0,
$$

which involves four basic operators $\left(I, S_{t}^{-}, S_{x}^{-}\right.$and $\left.S_{t}^{-} S_{x}^{-}\right)$. Therefore, the counter $m$ in the main loop in Box 1 will range from 1 to $4^{J}-1$, and all the calculation will be performed in base 4 rather than in base 3 . Similarly, the approximations (27) and (28) will require calculations in base 5 and base 8, respectively. The alternations needed in the coding are minor, but naturally the computational time associated with these improved approximations would increase dramatically. 
We note that when one uses a high-order Higdon NRBC, the discrete operator involved is of high-order even when the simplest formulas (13) are used to approximate the $x$-and $t$-derivatives. Thus, the importance of the improvements discussed above diminishes when $J$ increases. In fact, it is probably worthwhile to incorporate such improvements in the scheme only if a low-order condition (say, $J \leq 3$ ) is employed.

\section{Controlling the parameters}

The Higdon NRBCs involve the parameters $C_{j}$ which must be chosen. There are three approaches in this context:

(a) The user chooses the $C_{j}$ a priori in a manual manner based on an "educated guess". This is the procedure recommended in Higdon's papers [20-25].

(b) The $C_{j}$ are chosen automatically by the computer code as a preprocess.

(c) The $C_{j}$ are not constant, but are determined dynamically by the computer code. Namely, a value for $C_{j}$ is estimated for every grid point on the boundary at each time-step, from the solution in the previous time-steps.

We have adopted approach (b), which is automatic yet very inexpensive computationally. The algorithm we propose is described in Box 2. It is based on the maximum resolvable wavenumbers in the $x$ - and $y$-directions, and on the minimax formula [37] for choosing the $x$-component wavenumbers.

\section{Box 2. Algorithm, used as a preprocess, for determining the parameters $C_{j}$ in the Higdon NRBC.}

- Given the grid parameter $\Delta x$, estimate the maximum resolvable wavenumber $k$ in the $x$-direction. Assuming a maximum of 10 grid points per wavelength, a reasonable estimate is

$$
k_{\max }=\frac{\pi}{5 \Delta x} .
$$

- Choose $J-1$ values of $k$ from the interval $\left(0, k_{\max }\right)$. This is done using the symmetric minimax formula (based on the Chebyshev polynomial) proposed by Sommeijer et al. [37]

$$
k_{j}=\left[\frac{k_{\max }^{2}}{2}\left(1+\cos \left(\frac{2 j-1}{2(J-1)} \pi\right)\right)\right]^{1 / 2}, \quad j=1, \ldots, J-1 .
$$

- Given the grid parameter $\Delta y$, estimate the maximum resolvable wavenumber $k_{y}$ in the $y$-direction. Again assuming a maximum of 10 grid points per wavelength, a reasonable estimate is

$$
\left(k_{y}\right)_{\max }=\frac{\pi}{5 \Delta y} .
$$

- For each $k_{j}$, calculate the corresponding (and maximal in the $y$-direction) frequency $\omega_{j}$ from the dispersion relation (8)

$$
\omega_{j}=\sqrt{C_{0}^{2}\left[k_{j}^{2}+\left(k_{y}\right)_{\max }^{2}\right]+f^{2}} .
$$

- Calculate

$$
C_{j}=\frac{\omega_{j}}{k_{j}} \text { for } j=1, \ldots, J-1 .
$$

- Add the value $C_{0}$ (the minimum possible phase speed) to the $J-1$ values calculated above. These constitute the desired $J$ values $C_{j}$. 


\section{Numerical examples}

We first apply the new scheme to a simple test problem whose exact solution is synthesized a priori. We consider the waveguide problem described in Section 2 and illustrated in Fig. 1(a). We set $b=5, C_{0}=1$ and $f=0.5$. The boundary function $u_{\mathrm{W}}(y, t)$ on $\Gamma_{\mathrm{W}}$ and the initial conditions are those that correspond to a solution $u(x, y, t)$ which is a linear combination of three waves of the form (7), i.e.

$$
u=\sum_{m=1}^{3} A_{m} \cos \frac{n_{m} \pi y}{b} \cos \left(k_{m} x-\omega_{m} t\right) .
$$

The parameters chosen in (30) are $A_{m}=1,1,1 ; n_{m}=1,2,2 ; \omega_{m}=0.81,1.37,1.68$. This corresponds to the three phase velocities (obtained from (10)): $C_{x} / C_{0}=7.61,6.27,1.69$. The $k_{m}$ in (30) is obtained from the $\omega_{m}$ and the $n_{m}$ via the dispersion relation (8).

We introduce the artificial boundary $\Gamma_{\mathrm{E}}$ (see Fig. $1(\mathrm{~b})$ ) at $x_{\mathrm{E}}=5$. Thus, the computational domain $\Omega$ is a $5 \times 5$ square. In $\Omega$ we use a uniform grid with $21 \times 21$ points. We discretize the Klein-Gordon equation in $\Omega$ using the explicit central-difference FD interior scheme (25). On $\Gamma_{\mathrm{E}}$ we impose the Higdon NRBC implemented in its high-order form. The time-step size is $\Delta t=0.025$, which is smaller than the CFL limit and thus guarantees stability.

In Figs. 2(a)-(d), we plot the solution $u$ at the point $x=5, y=2.75$ (located on $\Gamma_{\mathrm{E}}$ ) as a function of time. In each of the four figures the exact solution is compared to a number of numerical solutions obtained with different
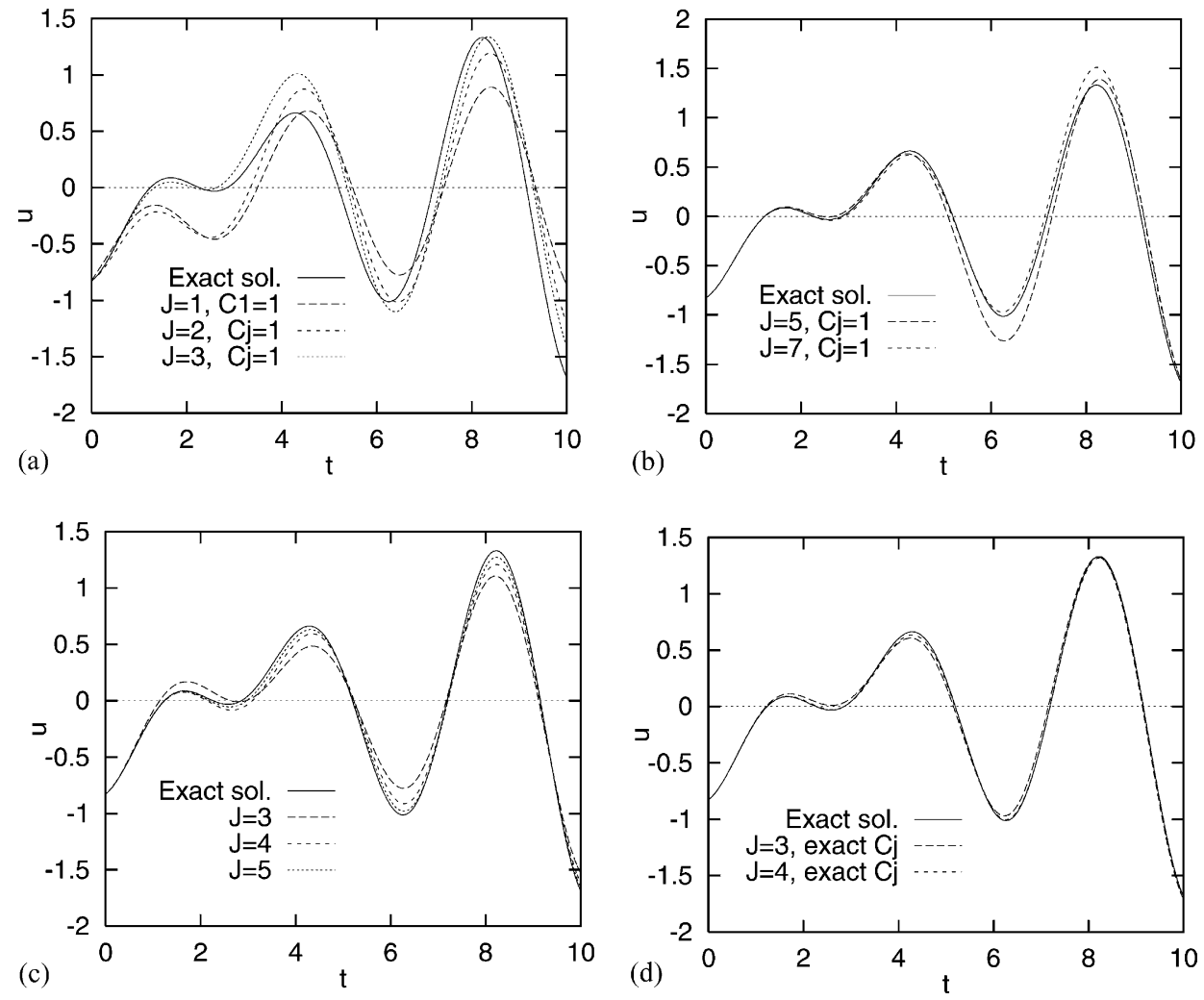

Fig. 2. Solution of the three-wave test problem: $u$ at the point $x=5, y=2.75$ (on $\Gamma_{\mathrm{E}}$ ) as a function of time. (a) Exact solution and the $H_{1}, H_{2}$ and $H_{3}$ solutions with $C_{j}=1$. (b) Exact solution and the $H_{5}$ and $H_{7}$ solutions with $C_{j}=1$. (c) Exact solution and the $H_{3}, H_{4}$ and $H_{5}$ solutions with automatically chosen $C_{j}$. (d) Exact solution and the $H_{3}$ and $H_{4}$ solutions with the exact $C_{j}$. 
NRBC schemes, namely with different choices of the order $J$ and the parameters $C_{j}$. First we choose $C_{j}=1$ for all $j$. Fig. 2(a) shows the $H_{1}, H_{2}$ and $H_{3}$ solutions. Their accuracy is poor, although the $H_{3}$ solution is significantly better than the other two. Fig. 2(b) shows the $\mathrm{H}_{5}$ and $\mathrm{H}_{7}$ solutions. The $\mathrm{H}_{7}$ solution is quite accurate in the entire time interval shown. Thus, if the $C_{j}$ 's are not specially chosen, we need the order of the Higdon NRBC to be as high as 7 for high accuracy.

Now we employ the procedure given by Box 2 to automatically choose the $C_{j}$ 's. Fig. 2(c) shows the resulting $H_{3}, H_{4}$ and $H_{5}$ solutions. We see that in this case the approach of the numerical solutions to the exact solution is monotone. Moreover, for $J=5$ we get about the same level of accuracy as we did with $J=7$ when all the $C_{j}$ had the value one (Fig. 2(b)). For additional reference, we show in Fig. 2(d) the $H_{3}$ solution obtained with the $C_{j}$ corresponding to the three phase velocities $C_{x}$ of the exact solution. It is about as accurate as the $H_{5}$ solution in Fig. 2(c). We also show the $H_{4}$ solution obtained with the exact $C_{1}, C_{2}, C_{3}$ and with $C_{4}=1$. The numerical solution is indistinguishable from the exact solution. In this case not only the NRBC is exact, but we gain additional accuracy on the boundary due to the increased order of the FD scheme.

This example demonstrates, albeit in a simplified setting, that the same level of accuracy obtained with parameter values $C_{j}$ that are well-estimated can be achieved with ill-chosen parameter values but with an increased order $J$. Of course, increasing the order to ensure high accuracy is computationally expensive, and therefore it is usually beneficial to use the algorithm given in Box 2.

We now consider another problem in the same waveguide, again with $b=5, C_{0}=1$ and $f=0.5$. The initial conditions are all zero, and the boundary function $u_{\mathrm{W}}$ on $\Gamma_{\mathrm{W}}$ is given by

$$
u_{\mathrm{W}}(y, t)= \begin{cases}\cos \left[\frac{\pi}{2 r}\left(y-y_{0}\right)\right] & \text { if }\left|y-y_{0}\right| \leq r \text { and } t \leq t_{0}, \\ 0 & \text { otherwise. }\end{cases}
$$

Thus, the wave source on the west boundary is a cosine function in $y$ with three parameters: its center location $y_{0}$, its width $r$, and its time duration $t_{0}$. We set $y_{0}=2.5, r=1.5$, and $t_{0}=0.5$. In contrast to the previous test problem, the solution of this problem involves an infinite number of modes and frequencies.

The computational parameters $x_{\mathrm{E}}, \Delta t$ and the grid are the same as in the previous example. However, here we use the Higdon NRBC $H_{4}$ on $\Gamma_{\mathrm{E}}$, with the four $C_{j}$ 's obtained automatically by using the procedure given in Box 2 . These turn out to be $C_{j} / C_{0}=1,1.45,1.75$ and 4.06 .

We compare the solution obtained by the new scheme with two other solutions:

- A solution obtained in the same domain, but with the Higdon NRBC $H_{1}$ on $\Gamma_{\mathrm{E}}$, using $C_{1}=5$. (Results obtained for $H_{1}$ with a smaller $C_{1}$ had a similar character, although less dramatic quantitatively.)

- A solution obtained in a domain twice as long, namely the domain $0 \leq x \leq 10,0 \leq y \leq 5$, using a $42 \times 21$ grid with the same resolution. During the simulation time the wave generated on $\Gamma_{\mathrm{W}}$ does not reach the remote (east) boundary of this large domain, and thus the issue of spurious reflection is avoided altogether, regardless of the boundary condition used on the remote boundary. Hence this will serve as a "reference solution" which is exact as far as the boundary condition treatment is concerned.

Fig. 3(a) shows the three solutions at time $t=4$. In this and the next figures, the top plot is that of the reference solution, the middle plot corresponds to the solution obtained by the new scheme with the $H_{4} \mathrm{NRBC}$, and the lower plot describes the $H_{1}$ solution. Both the colors and the contour lines represent values of $u$. At time $t=4$ the main bulk of the wave packet generated on $\Gamma_{\mathrm{W}}$ has not reached the boundary $\Gamma_{\mathrm{E}}$ yet, and hence all three plots are similar.

Fig. 3(b) shows the three solutions at time $t=6$. The wave packet has already passed the boundary $\Gamma_{\mathrm{E}}$. The $H_{4}$ solution is indistinguishable from the reference solution, whereas in the $H_{1}$ solution spurious reflection is evident. Figs. 3(c) and (d) correspond to times $t=8$ and 10, respectively. The reflected wave moves backward in the $H_{1}$ solution and pollutes the entire computational domain. On the other hand, the $H_{4}$ solution exhibits the wave traces which are also present in the reference solution.

Finally, we repeat this experiment while increasing the dispersion parameter by a factor of 20 , i.e., we take $f=10$. The group velocity decreases with increasing dispersion; hence the wave packet will move much more slowly now. 

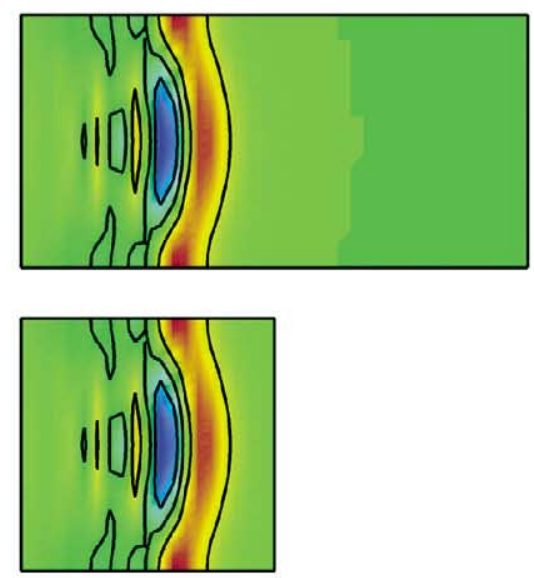

(a)
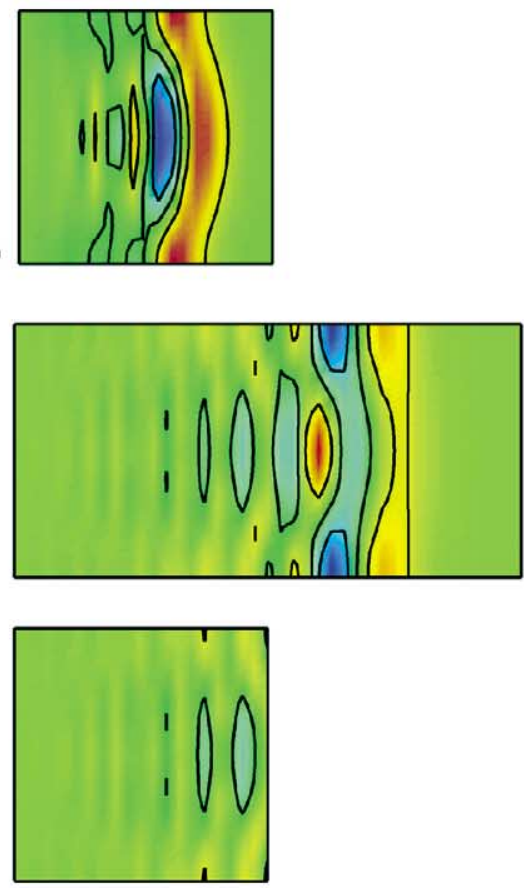

(c)

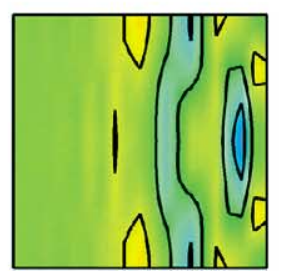

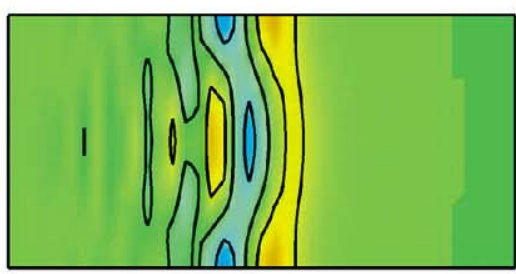

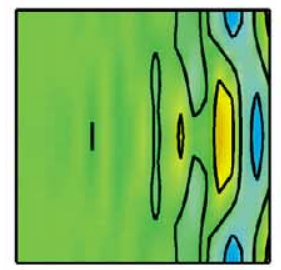

(b)
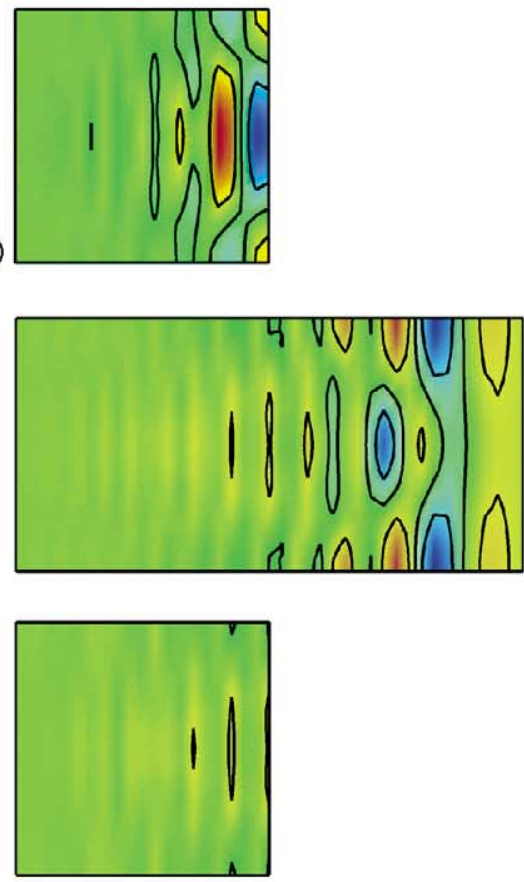

(d)

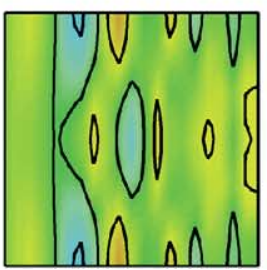

Fig. 3. Solution of the west-source problem, dispersion parameter $f=0.5$. Top plot-reference solution, middle plot $-H_{4}$ solution, lower plot- $H_{1}$ solution. Times: (a) $t=4$, (b) $t=6$, (c) $t=8$, (d) $t=10$. 

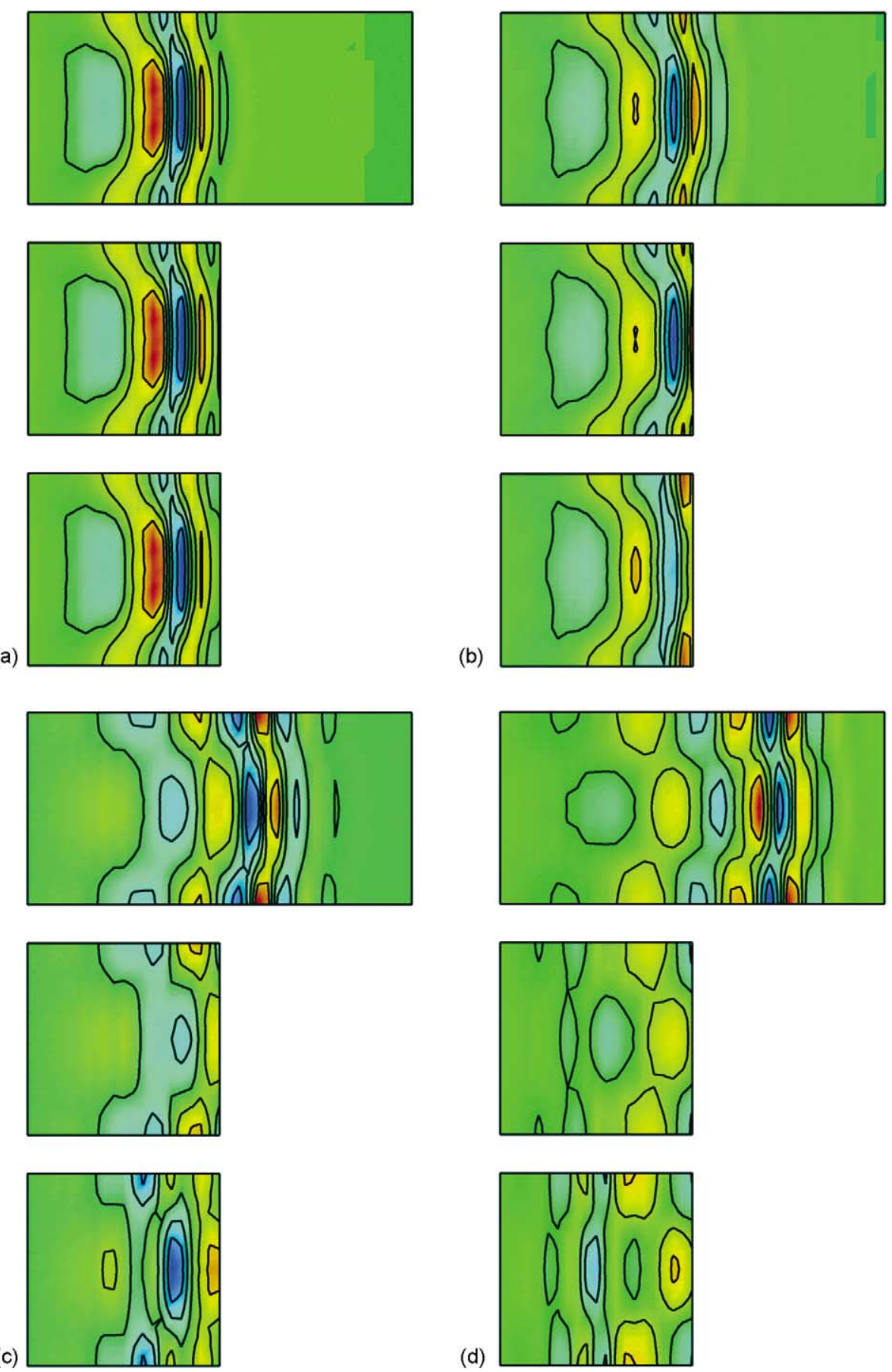

Fig. 4. Solution of the west-source problem, dispersion parameter $f=10$. Top plot-reference solution, middle plot $-H_{4}$ solution, lower plot- $H_{1}$ solution. Times: (a) $t=12.5$, (b) $t=15$, (c) $t=19$, (d) $t=22.5$. 
Also, the solution is expected to be much "richer", being composed of many waves with different frequencies and phase speeds. Indeed, this is seen in Fig. 4(a), which illustrates the solution at time $t=12.5$. The wave has just reached the boundary $\Gamma_{\mathrm{E}}$, and the beginning of spurious reflection in the $H_{1}$ solution is apparent. Figs. 4(b), (c) and (d) show the solutions at times $t=15,19$ and 22.5, respectively. Again the $H_{1}$ solution exhibits spurious reflection, while the $H_{4}$ solution performs very well in this high-dispersion case too.

\section{Concluding remarks}

In this paper we have presented a new numerical procedure based on FDs, which allows the use of the Higdon NRBCs up to an arbitrarily high-order. The scheme is coded once and for all for any order; the order of the scheme is simply an input parameter. This adds an important computational tool for use in the solution of infinite domain time-dependent wave problems. Moreover, due to the generality of the Higdon NRBCs it is possible to use this tool for problems with dispersive and layered media. We have focused on dispersive waves in this paper, although the scheme is effective in the non-dispersive case as well. We have demonstrated that the high-order scheme, with the automatically chosen parameters, performs very well in both the low and high dispersion regimes.

Related future work will include the adaptation of the proposed approach to more complicated configurations, such as exterior problems with a rectangular artificial boundary $\mathcal{B}$, and three-dimensional problems. It should be remarked that no difficulties are expected in the case where $\mathcal{B}$ has corners. The Higdon NRBCs do not involve mixed spatial derivatives, and thus the corner grid point can arbitrarily be associated with one of the two straight edges that meet at this corner, and can be treated just as any other grid point on this chosen edge.

In addition, the high-order Higdon NRBCs will be applied to the SWEs. The shallow water model serves as an important test-bed for more complicated models in meteorology [38]. It would be interesting, among other things, to test the performance of the high-order Higdon NRBCs when the non-linear SWEs are used in the computational domain $\Omega$.

An alternative formulation of the Higdon NRBCs is currently under development. In this formulation some auxiliary variables are introduced on the artificial boundary, in order to eliminate all the high-order derivatives. This form of the NRBC has the advantages that after discretization it involves only degrees of freedom on the boundary $\mathcal{B}$ itself, that no high-order discrete schemes are needed, and that the history of the solution does not have to be stored. As a result, it is more amenable, compared to the formulation presented in this paper, for incorporation in a Finite Element scheme. This alternative formulation will be reported in a future publication.

\section{Acknowledgements}

This work was supported by the US National Research Council (NRC), the Office of Naval Research (ONR), and the Naval Postgraduate School (NPS).

\section{References}

[1] D. Givoli, Numerical Methods for Problems in Infinite Domains, Elsevier, Amsterdam, 1992.

[2] D. Givoli, Non-reflecting boundary conditions: a review, J. Comput. Phys. 94 (1991) 1-29.

[3] S.V. Tsynkov, Numerical solution of problems on unbounded domains: a review, Appl. Numer. Math. 27 (1998) $465-532$.

[4] D. Givoli, Exact representations on artificial interfaces and applications in mechanics, Appl. Mech. Rev. 52 (1999) $333-349$.

[5] B. Engquist, A. Majda, Radiation boundary conditions for acoustic and elastic calculations, Comm. Pure Appl. Math. 32 (1979) $313-357$.

[6] A. Bayliss, E. Turkel, Radiation boundary conditions for wave-like equations, Comm. Pure Appl. Math. 33 (1980) $707-725$.

[7] J.B. Keller, D. Givoli, Exact non-reflecting boundary conditions, J. Comput. Phys. 82 (1989) 172-192.

[8] D. Givoli, J.B. Keller, Non-reflecting boundary conditions for elastic waves, Wave Motion 12 (1990) 261-279.

[9] J.P. Bérenger, A perfectly matched layer for the absorption of electromagnetic waves, J. Comput. Phys. 114 (1994) 185-200. 
[10] F. Collino, High order absorbing boundary conditions for wave propagation models. Straight line boundary and corner cases, in: R. Kleinman, et al. (Eds.), Proceedings of the Second International Conference on Mathematical and Numerical Aspects of Wave Propagation, SIAM, Delaware, 1993, pp. 161-171.

[11] M.J. Grote, J.B. Keller, Nonreflecting boundary conditions for time dependent scattering, J. Comput. Phys. 127 (1996) $52-65$.

[12] M.J. Grote, J.B. Keller, Nonreflecting boundary conditions for elastic waves, SIAM J. Appl. Math. 60 (2000) 803-819.

[13] I.L. Sofronov, Conditions for complete transparency on the sphere for the three-dimensional wave equation, Russ. Acad. Dci. Dokl. Math. 46 (1993) 397-401.

[14] T. Hagstrom, S.I. Hariharan, A formulation of asymptotic and exact boundary conditions using local operators, Appl. Numer. Math. 27 (1998) 403-416.

[15] M.N. Guddati, J.L. Tassoulas, Continued-fraction absorbing boundary conditions for the wave equation, J. Comput. Acoust. 8 (2000) $139-156$.

[16] D. Givoli, High-order non-reflecting boundary conditions without high-order derivatives, J. Comput. Phys. 170 (2001) 849-870.

[17] D. Givoli, I. Patlashenko, An optimal high-order non-reflecting finite element scheme for wave scattering problems, Int. J. Numer. Meth. Eng. 53 (2002) 2389-2411.

[18] J. Pedlosky, Geophys. Fluid Dyn., Springer, New York, 1987.

[19] I.M. Navon, B. Neta, M.Y. Hussaini, A perfectly matched layer formulation for the nonlinear shallow water equations models: the split equation approach, in press.

[20] R.L. Higdon, Radiation boundary conditions for dispersive waves, SIAM J. Numer. Anal. 31 (1994) 64-100.

[21] R.L. Higdon, Absorbing boundary conditions for difference approximations to the multi-dimensional wave equation, Math. Comput. 47 (1986) 437-459.

[22] R.L. Higdon, Numerical absorbing boundary conditions for the wave equation, Math. Comput. 49 (1987) 65-90.

[23] R.L. Higdon, Radiation boundary conditions for elastic wave propagation, SIAM J. Numer. Anal. 27 (1990) 831-870.

[24] R.L. Higdon, Absorbing boundary conditions for elastic waves, Geophysics 56 (1991) 231-241.

[25] R.L. Higdon, Absorbing boundary conditions for acoustic and elastic waves in stratified media, J. Comput. Phys. 101 (1992) $386-418$.

[26] R.A. Pearson, Consistent boundary conditions for the numerical models of systems that admit dispersive waves, J. Atmos. Sci. 31 (1974) $1418-1489$.

[27] I. Orlanski, A simple boundary condition for unbounded hyperbolic flows, J. Comput. Phys. 21 (1976) 251-269.

[28] W.H. Raymond, H.L. Kuo, A radiation boundary condition for multi-dimensional flows, Q.J.R. Meteorol. Soc. 110 (1984) $535-551$.

[29] M.J. Miller, A.J. Thorpe, Radiation conditions for the lateral boundaries of limited-area numerical models, Q.J.R. Meteorol. Soc. 107 (1981) 615-628.

[30] J.B. Klemp, D.K. Lilly, Numerical simulation of hydrostatic mountain waves, J. Atmos. Sci. 35 (1978) $78-107$.

[31] M. Wurtele, J. Paegle, A. Sielecki, The use of open boundary conditions with the storm-surge equations, Mon. Wea. Rev. 99 (1971) 537-544.

[32] R.M. Hodur, The naval research laboratory's coupled ocean/atmosphere mesoscale prediction system (COAMPS), Mon. Wea. Rev. 125 (1997) 1414-1430.

[33] X. Ren, K.H. Wang, K.R. Jin, Open boundary conditions for obliquely propagating nonlinear shallow-water waves in a wave channel, Comput. Fluids 26 (1997) 269-278.

[34] T.G. Jensen, Open boundary conditions in stratified ocean models, J. Mar. Syst. 16 (1998) 297-322.

[35] F.S.B.F. Oliveira, Improvement on open boundaries on a time dependent numerical model of wave propagation in the nearshore region, Ocean Eng. 28 (2000) 95-115.

[36] J.C. Strikwerda, Finite Difference Schemes and Partial Differential Equations, Wadsworth and Brooks, Pacific Grove, CA, 1989.

[37] B.P. Sommeijer, P.J. van der Houwen, B. Neta, Symmetric linear multistep methods for second order differential equations with periodic solutions, Appl. Numer. Math. 2 (1986) 69-77.

[38] D.R. Durran, Numerical Methods for Wave Equations in Geophysical Fluid Dynamics, Springer, New York, 1999. 